\title{
Predictable or fateful-a short guide for how to anticipate risk to keep the incidence of aortoesophageal fistulation to a minimum
}

\author{
Martin Czerny, MD, MBA
}

\author{
From the University Heart Center Freiburg-Bad Krozingen, Südring, Germany. \\ Disclosures: Author has nothing to disclose with regard to commercial support. \\ Received for publication Sept 9, 2017; accepted for publication Sept 9, 2017; available ahead of print Oct 10, \\ 2017. \\ Address for reprints: Martin Czerny, MD, MBA, Albert Ludwigs University Freiburg-Faculty of Medicine, Uni- \\ versity Heart Center Freiburg-Bad Krozingen, Südring 15 79189, Germany (E-mail: martin.czerny@ \\ universitaets-herzzentrum.de). \\ J Thorac Cardiovasc Surg 2018;155:41-2 \\ 0022-5223/ $\$ 36.00$ \\ Copyright (C) 2017 by The American Association for Thoracic Surgery \\ https://doi.org/10.1016/j.jtcvs.2017.09.043
}

Aortoesophageal fistulations (AEFs) often are seen as fateful, but many of them are predictable, in particular in patients who have undergone previous thoracic endovascular aortic repair (TEVAR) under certain given conditions. To better understand the mechanisms behind their formation, a short excursion into posterior mediastinal anatomy is helpful.

The esophagus per se has no serosa, which predisposes it to a kind of vulnerability when affected by adjacent processes, in particular pressure via either neighboring organs in case of large chronic aneurysmal formation or by hematoma formation during (contained) ruptures in acute aortic syndromes. Both mechanisms lead to a further reduction in native blood supply, which often is tolerated and compensated but after a certain threshold will lead to local tissue necrosis and finally fistula formation. This process is rarely an acute one but occurs slowly, with a median duration of 3 months between the acute event and AEF formation, according to the European Registry of Endovascular Complications after TEVAR, and may even take longer, as reported here. ${ }^{1,2}$

What this study nicely shows, thereby confirming previous reports, is that the pattern behind AEF formation seems to be largely consistent. Let us focus on diameter and aortic tissue behavior after TEVAR. The very patients who developed native fistulation had a large aneurysmal sac and most likely were not aware of their underlying thoracic aortic pathology, so anticipation was not possible here, but the message for a physician advising patients in aortic centers when to indicate treatment should be awareness that in addition to rupture, AEF is one of the rare potential collateral injuries in the natural course of the disease as is the case in infrarenal aneurysm formation and the rare occurrence of aortoduodenal fistulation, which also seems to be a metachronous process in the majority of patients.

In clinical scenarios in which there is time to anticipate and to reflect on the most appropriate treatment option, the size of the aneurysm should play a substantial role in advocating for classical surgery or TEVAR. One of the

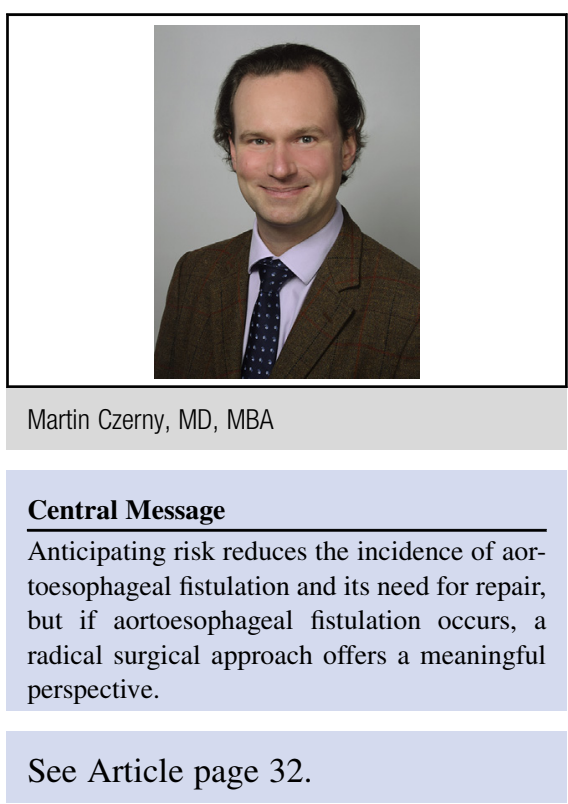

mechanisms behind AEF formation seems to be additional pressure exertion onto the esophagus by TEVAR, as the radial force of the stent-graft amplifies the alreadyexistent natural pressure exerted by the disease process per se. In case of persisting or recurring endoleak formation, this effect is even more pronounced, as could be shown in patients who developed aortobronchial/pulmonary fistulations - both major airway as well as parenchymatous fistulations. ${ }^{3}$ Therefore, we need to think twice before we recommend TEVAR in very large aneurysms because of the remaining risk of secondary AEF or aortobronchial/pulmonary fistulation.

However, as the authors have shown, classical surgery also is not free from the remaining risk of secondary AEF. One of our main conceptual approaches in classical thoracoabdominal replacement is to remain within the aneurysmal sac during repair as much as possible and to keep extra-aortic tissue dissection to a minimum, focusing on the very areas in which sequential clamping for repair will be applied as to not affect major blood supply to an already ischemia-susceptible organ, as the esophagus is. Finally, surgical stitches, in particular when performing the proximal anastomosis at the level of the distal aortic arch in type I or type II repairs, warrant the prevention of unnecessary deep needle penetration of aortic tissue because of esophageal proximity. 
Finally, the question of the most adequate repair has to be answered. The authors have shown nicely that TEVAR may well serve as a bridge to definitive therapy and should not be seen as a definite treatment option because of the continuing propagation of the infective process, inevitably leading to tertiary limitations. ${ }^{4}$ The futility of solutions by halves also has been shown in the European Registry of Endovascular Complications cohort, be it conservative therapy or isolated esophagectomy, leaving the infected alloplastic vascular material in place. ${ }^{3,4}$ The authors used rifampicinsoaked Dacron prostheses, achieving excellent results in their series. The trade-off of this approach is the need for patients to remain on life-long antibiotic therapy.

In our experience, self-made pericardial tube grafts used as neoaortas perform very well, with the potential to withdraw antibiotic therapy after several months with the exception of fungal infections, for which we also maintain lifelong antifungal therapy. ${ }^{5}$ Finally, the authors remind us to use an important adjunct, omentoplasty, to create a biologic seal, which is definitely a major component to success, in particular when cavitation would leave room for fluid accumulation, creating the conditions for persisting or recurring infection.
In summary, this report, by its excellent results, should motivate the surgical community to offer radical treatment to patients with AEF, as it remains the only meaningful perspective in a very complex scenario. Attention to detail has to be claimed already at the time when recommending the most suitable treatment modality to anticipate risk and to reduce the remaining risk of AEF to a minimum.

\section{References}

1. Yamazato T, Nakamura T, Abe N, Yokawa K, Ikeno Y, Koda Y, et al. Surgical experience of aorto-esophageal fistula. J Thorac Cardiovasc Surg. 2018;155: 32-40.

2. Czerny M, Eggebrecht H, Sodeck G, Weigang E, Livi U, Verzini F, et al. New insights regarding the incidence, presentation and treatment options of aortooesophageal fistulation after thoracic endovascular aortic repair: the European Registry of Endovascular Aortic Repair Complications. Eur J Cardiothorac Surg. 2014;45:452-7.

3. Czerny M, Reser D, Eggebrecht H, Janata K, Sodeck G, Etz C, et al. Aorto-bronchial and aorto-pulmonary fistulation after thoracic endovascular aortic repair: an analysis from the European Registry of Endovascular Aortic Repair Complications. Eur J Cardiothorac Surg. 2015;48:252-7.

4. Stellmes A, von Allmen R, Derungs U, Dick F, Makaloski V, Do DD, et al. Thoracic endovascular aortic repair as emergency therapy despite suspected aortic infection. Interact Cardiovasc Thorac Surg. 2013;16:459-64.

5. Czerny M, von Allmen R, Opfermann P, Sodeck G, Dick F, Stellmes A, et al. Selfmade pericardial tube-graft: a new surgical concept for treatment of graft infections after thoracic and abdominal aortic procedures. Ann Thorac Surg. 2011; 92:1657-62. 